\title{
The impact of trainee involvement on outcomes in low dose rate brachytherapy for prostate cancer
}

\author{
Talha Shaikh, MD ${ }^{1}$, Lora Wang, MD ${ }^{1}$, Karen Ruth, $\mathbf{M S}^{2}$, Mark Hallman, MD, PhD ${ }^{1}$, David Y. \\ Chen, MD ${ }^{3}$, Richard E. Greenberg, MD ${ }^{3}$, Jinsheng Li, PhD ${ }^{1}$, Kevin Crawford, BS ${ }^{1}$, and Eric \\ M Horwitz, MD ${ }^{1}$ \\ ${ }^{1}$ Departments of Radiation Oncology, Fox Chase Cancer Center, Philadelphia, PA \\ ${ }^{2}$ Departments of Biostatistics, Fox Chase Cancer Center, Philadelphia, PA \\ ${ }^{3}$ Departments of Surgical Oncology, Fox Chase Cancer Center, Philadelphia, PA
}

\section{Abstract}

Purpose-To determine the impact of fellow, resident, or medical student (MS) involvement on outcomes in patients undergoing permanent Iodine-125 prostate seed implant.

\begin{abstract}
Methods and Materials-The study population consisted of men with clinically localized low-I intermediate-risk prostate cancer treated with low dose rate permanent interstitial brachytherapy. Cases were stratified according to resident, fellow, MS, or attending involvement. Outcomes were compared using ANOVA, logistic regression, and log rank tests.
\end{abstract}

\begin{abstract}
Results-A total of 291 patients were evaluated. Fellows, residents, and MS were involved in $47(16.2 \%), 231(79.4 \%)$, and 34(11.7\%) cases, respectively. Thirteen (4.4\%) cases were completed by an attending physician alone. There was no difference in FFBF when comparing the resident, fellow, or attending alone groups ( $\mathrm{p}=0.10$ ). There was no difference in V100 (volume of the prostate receiving $100 \%$ of the prescription dose) outcomes when comparing resident cases to fellow cases $(\mathrm{p}=0.72)$ or attending alone cases $(\mathrm{p}=0.78)$. There was no difference in D90 (minimum dose covering $90 \%$ of the post-implant volume) outcomes when comparing resident cases to fellow cases $(\mathrm{p}=0.74)$ or attending alone cases $(\mathrm{p}=0.58)$. When examining treatment toxicity, fellow cases had higher rates of acute grade $2+\mathrm{GU}$ toxicity $(\mathrm{p}=0.028)$. With the exception of higher urethra D90 among PGY 2-3 cases ( $\mathrm{p}=0.02$ ), dosimetric outcomes were similar to cases with PGY4-5 resident participation. There was no difference in outcomes for cases with and without MS participation.
\end{abstract}

Conclusions-Interstitial prostate seed implants can be safely performed by trainees with appropriate supervision. Hands-on brachytherapy training is effective and feasible for trainees.

Correspondence should be directed to: Eric M. Horwitz, MD, Department of Radiation Oncology, Fox Chase Cancer Center, 333 Cottman Avenue, Philadelphia, PA 19111, eric.horwitz@fccc.edu, Telephone: 215-728-2995, Fax: 215-728-2868.

Conflicts of Interest: None

Publisher's Disclaimer: This is a PDF file of an unedited manuscript that has been accepted for publication. As a service to our customers we are providing this early version of the manuscript. The manuscript will undergo copyediting, typesetting, and review of the resulting proof before it is published in its final citable form. Please note that during the production process errors may be discovered which could affect the content, and all legal disclaimers that apply to the journal pertain. 


\section{Keywords}

LDR; prostate cancer; resident; medical student; training; brachytherapy

\section{Introduction}

Low dose rate (LDR) Iodine-125 (I-125) prostate brachytherapy is an established standard of care treatment in the management of patients with low- to intermediate-risk prostate cancer. Recent studies have demonstrated a decreasing trend in the utilization of brachytherapy likely due to the introduction of new technologies, changes in economic incentive and increasing use of active surveillance. [1] As a result, there has been a significant reduction in resident-reported brachytherapy experience during training [2].

Brachytherapy procedures provide an important learning opportunity for radiation oncology resident physicians because of their complexity and intricacy. Therefore, they are an important part of radiation oncology residency. Maintaining patient safety is a critical aspect of these procedures due to the proximity of radioactive sources to sensitive normal organs and the potential for significant complications. While simulation and training models exist, hands on experience remains the best means for learning these procedures. Patient trepidation regarding trainee involvement is common due to the belief that this may adversely impact treatment outcomes.

In surgical specialties, multiple series have examined the impact of resident or fellow involvement on outcomes with conflicting reports [3, 4]. To our knowledge, there are no studies that have addressed the possible impact of trainee involvement on outcomes in patients undergoing LDR prostate brachytherapy. The objective of this study is to examine how brachytherapy outcomes are affected by resident, fellow, or medical student involvement.

\section{Materials and Methods}

\section{Patients}

The study population consisted of men with low- to intermediate-risk (T1-2, N0, M0) prostate cancer treated with I-125 LDR prostate brachytherapy alone at a National Cancer Institute-designated Comprehensive Cancer Center. Patient demographics, tumor characteristics, and treatment-related information were entered prospectively into an Institutional Review Board-approved database that was maintained and updated by data managers. The collection, storage, and retrieval of data were all done in compliance with the hospital's Institutional Review Board and the Health Insurance Privacy and Portability Act. Risk groups were stratified per National Comprehensive Cancer Network (NCCN) criteria, with low risk comprised of patients with clinical stage $\leq \mathrm{T} 2 \mathrm{a}$, PSA $<10 \mathrm{ng} / \mathrm{ml}$ and Gleason score $\leq 6$. Intermediate risk patients consisted of clinical stage T2b-T2c, PSA 10-20 ng/ml and Gleason score 7. At our institution, high-risk patients are not offered I-125 monotherapy per NCCN guidelines [5]. 
In addition to clinical criteria, patients offered I-125 prostate seed implants at our institution must meet criteria set forth by the American Brachytherapy Society [6]. Relative contraindications include large prostate volumes $\left(>60 \mathrm{~cm}^{3}\right)$, high international prostate symptom score (>20), history of pelvic radiation, large median lobe, or transurethral resection defects. Patients are also evaluated by a board-certified anesthesiologist and urologic oncologists to evaluate for operative risk prior to the procedure.

\section{Treatment technique/Trainee Involvement}

Planning and procedure technique have been previously described [7, 8]. All patients were treated using a stranded technique in which sleeves containing seeds and spacers were matched to the real-time plan and loaded into the applicator needles. The applicator needles were sequentially inserted under ultrasound guidance using sagittal and axial views. Fluoroscopic examination was performed midway and at the completion of treatment to confirm that no seeds are placed in the bladder or urethra.

At our institution, residents or fellows routinely first assist in procedures while rotating medical students are second assist. In general, only one resident or fellow is typically involved and medical students are not the only trainee involved. Trainees are closely involved in all aspects of the procedure. This includes driving the ultrasound probe and aligning the prostate position and grid. Typically trainees place needles which are then verified by the attending physician for placement. If there are multiple trainees involved, participants rotate placing needles which are verified by an attending after each placement. If the trainee is unable to successfully place a needle after multiple attempts, the attending physician will then place the needle. Briefly, the number and activity of I-125 seeds for each patient is calculated using an MRI-generated, physician-contoured volume with experience in the procedure and reviewed with at least one other experienced attending physician. All procedures are performed under the supervision of a board-certified radiation oncologist with experience in I-125 prostate implants and all trainees are required to complete the appropriate radiation safety related training.

During the procedure, patients were placed under general anesthesia in extended dorsal lithotomy position. Intraoperative planning and seed placement is under real-time ultrasound guidance using physician-generated contours of the prostate. A 3- to 5-mm anterior and lateral expansion was applied to the prostate volume to generate the planning target volume used for treatment. A total of 145 Gray (Gy) is prescribed to cover 100\% of the prostate volume. Per ABS guidelines, an acceptable plan must achieve a D90 > 145 Gy and V100 > $90 \%$. Midway through treatment and at the end of treatment, patients undergo fluoroscopic examination to document proper seed placement. Rigid cystoscopy is performed by the urologic oncologist to ensure the absence of needles, seeds or injury in the urethra or bladder at the end of the procedure. Approximately four hours after the implant, patients have the foley catheter removed and undergo post-implant CT and MRI to document baseline postimplant dosimetry prior to discharge. Patients return in approximately 3-4 weeks after implant to repeat the CT and MRI and generate the post-implant dosimetry for confirmation of dosimetric quality indicators and ensure seed stability and prostate dose coverage. 
Trainees complete post-implant dosimetry contouring which the treating physician subsequently verifies.

\section{Statistical Analysis}

Trainee involvement was identified by reviewing operative reports from brachytherapy cases. Cases were stratified according to resident (PGY 2-5), fellow (PGY 6+), medical student, or attending only involvement. Acute toxicity and dosimetric outcomes were compared across subgroups (resident versus fellow versus attending; resident PGY training year; medical student versus no medical student) using ANOVA and logistic regression models. Covariates adjusted in the model-included transition to sleeves, attending physician effect, and intraoperative prostate volume. Freedom from biochemical failure (FFBF) was estimated using the Kaplan-Meier product limit method where patients without biochemical failure were censored at the date of their most recent PSA determination. Log rank tests were used to evaluate differences in FFBF and late toxicity between groups. Biochemical failure was defined according to the Phoenix definition, $2 \mathrm{ng} / \mathrm{ml}$ rise above the PSA nadir [9]. Toxicity was measured using our institutional grading system and has been previously described [10, 11]. Statistical analyses were done using SAS 9.3 (SAS Institute, Cary, NC).

\section{Results}

\section{Patient Characteristics}

There were a total of 291 men with a median follow-up of 38.4 months (range 6-124). The majority of patients had low risk features including T1c disease $(n=258)$, Gleason 6 disease $(\mathrm{n}=284)$ and PSA $<10 \mathrm{ng} / \mathrm{mL}(\mathrm{n}=275)$. At least one post-implant PSA value was available in 98.9\% ( $\mathrm{n}=274)$ patients. Fellows, residents, and medical students were involved in 47 (16.2\%), 231 (79.4\%), and 34 (11.7\%) cases, respectively. Thirteen (4.4\%) cases were completed by an attending physician alone. There were less patients with $2 \mathrm{~T} 2 \mathrm{a}$ in the resident cohort versus the attending alone or fellow cohort ( $\mathrm{p}=0.04)$. Otherwise there was no significant difference between groups in terms of patient or treatment characteristics (Table $1 \&$ Table 2).

\section{Biochemical Failure}

There were a total of 8 biochemical failures with 5 (2.2\%) occurring in the resident group, 1 $(2.2 \%)$ in the fellows group, and $2(15.4 \%)$ in the attending alone group. The FFBF rate at 36 months for the residents, fellows, and attending alone groups were $95 \%, 95 \%$, and $90 \%$, respectively. After adjusting for T-stage there was no significant difference in FFBF rates between treatment groups $(\mathrm{p}=0.10)$. When examining cases with medical student involvement, there were $2(6 \%)$ failures in cases with medical student involvement and 6 (2.3\%) failures in cases without medical student involvement. The 36 month FFBF rate for the medical student versus non-medical student cases was $97 \%$ and $98 \%$, respectively. There was no significant difference in FFBF between treatment groups ( $\mathrm{p}=0.81$ ).

\section{Dosimetric Outcomes}

The median V100 for resident, fellow, and attending alone cases was 150.4 (range 90.6-208.1), 137.9 (range 64.9-219.6), and 157.1 (range 121.1-211.8), respectively. The 
median V150 for resident, fellow, and attending alone cases was 57.7 (range 24.4-95.8), 54.3 (range 23.1-90.8), and 58.5 (range 32.4-86.2), respectively. The median V200 for resident, fellow, and attending alone cases was 25 (range 7.6-65), 24.9 (range 9.8-52.6), and 24.4 (range 13.7-58.2), respectively. There was no difference in V100 outcomes among resident cases compared to fellow cases (OR 0.87, 95\% CI 0.40-1.90) or attending alone cases (OR 1.19, 95\% CI 0.35-4.03). The median D90 for resident, fellow, and attending alone cases was 91.7 (range 73.7-100), 87.6 (range 58.4-99.7), and 93.5 (range 77.9-100), respectively. Similarly, there was no difference in D90 outcomes among resident cases compared to fellow cases $(0.80,95 \%$ CI $0.36-1.77)$ or attending alone cases (OR $1.2395 \%$ CI 0.35-4.48). Further data is demonstrated in table 3. The PGY 2-3 group had a higher urethra D90, otherwise there was no difference between subgroups according to urethra D10, D30, or D90 outcomes (Figure 1A).

There were no differences in V100 outcomes among PGY 2 versus PGY 3-5 cases (OR 1.08 95\% CI 0.60-1.92), PGY 2-3 versus PGY 4-5 cases (OR 0.88 95\% CI 0.46-1.66), or PGY 2-4 versus PGY 5 cases (OR 0.66 95\% CI 0.22-2.00). Similarly, there were no differences in D90 outcomes among PGY 2 versus PGY 3-5 cases (OR 0.94 95\% CI 0.51-1.75), PGY 2-3 versus PGY 4-5 cases (OR 0.66 95\% CI 0.33-1.34), or PGY 2-4 versus PGY 5 cases (OR $0.395 \%$ CI 0.07-1.40). The mean urethra D90 was higher ( $\mathrm{p}=0.02$, Figure $1 \mathrm{~B}$ ) among cases with junior resident (PGY 2-3) participation compared to cases involving senior residents (PGY4-5). There was no other difference in urethra dosimetric endpoints among junior resident cases compared to senior resident cases.

There was no difference in V100 outcomes among cases with medical student involvement compared to cases without medical student involvement (OR $0.9795 \%$ CI 0.40-1.99). There was no difference in D90 outcomes among medical student versus non-medical student cases (OR 1.72 95\% CI 0.66-4.28). There was no impact on urethra dosimetric endpoints according to medical student involvement (Figure 1C).

\section{Treatment Toxicity}

A total of 117 (40.2\%) patients experienced acute grade 2+ GU toxicity and 8 (2.7\%) patients experienced acute grade $2+$ GI toxicity. When comparing groups, cases with fellow involvement had higher rates of acute grade $2+\mathrm{GU}$ toxicity versus the attending alone or resident groups (Fellow-59.5\%, Attending 46.2\%, Resident 38.5\%, $\mathrm{p}=0.028$ ). There was no difference in acute grade $2+$ GI toxicity $(\mathrm{p}=0.742)$. When examining late toxicity, there was no significant difference in grade $2+\mathrm{GI}(\mathrm{p}=0.974)$ or $\mathrm{GU}(\mathrm{p}=0.173)$ toxicity. There was no difference in acute grade $2+\mathrm{GU}(\mathrm{p}=1.00)$ or grade $2+\mathrm{GI}(0.855)$ toxicity according to medical student involvement. Similarly there was no difference in late grade $2+\mathrm{GU}$ $(\mathrm{p}=0.113)$ or $\mathrm{GI}(\mathrm{p}=0.997)$ according to medical student involvement.

\section{Discussion}

Educating and evaluating resident physicians while maintaining high quality treatment and patient safety is an important responsibility of attending physicians at academic medical centers. Designing and implementing training curricula is complex due to the desire to improve education while reducing errors. Our series demonstrates that under experienced 
supervision, trainee involvement does not have a significant impact on dosimetric, biochemical or clinical outcomes in patients undergoing I-125 permanent interstitial prostate implants for low- to intermediate-risk prostate cancer. To our knowledge, this is the first series examining the impact of resident, fellow, or medical student involvement on real world outcomes in the radiation oncology setting. Interstitial prostate brachytherapy remains an important hands-on learning experience and should continue to be provided to all radiation oncology resident physicians.

Achieving a D90 > 145 Gy and a V100 > 90\% are important surrogates of high quality implants and for biochemical control as demonstrated in multiple series [12, 13]. In the present series, D90 and V100 outcomes were similar regardless of resident or fellow involvement, PGY trainee year, or medical student involvement. Not surprisingly, there was also no difference in FFBF rates amongst the treatment groups after controlling for T-stage. Overall, there were only 8 biochemical failures in the entire cohort confirming the excellent efficacy of this treatment approach. Performing high quality implants under experienced supervision remains an important learning experience for radiation oncology trainees.

When examining treatment related toxicity, patients in the fellow groups had higher rates of acute grade 2+ GU toxicity although there was no other difference in acute or late treatment related side effects. Our results suggest that close monitoring by an attending is essential for quality implants. Due to the extensive experience of fellows, they are often given more independence during brachytherapy procedures. Our results underline the importance of close attending supervision during procedures. In addition, our results confirm the importance of case volume and case load on improving treatment technique. Furthermore, there was a higher mean urethra D90 when comparing lower year (PGY 2-3) versus higher year (PGY 4-5) cases. There is no well-defined urethra D90 cut-point identified so it is unclear if this difference is clinically meaningful. All other urethra dosimetric endpoints were similar among the treatment cohorts. The improvement in urethra dosimetric outcomes with trainee year suggests that case volume may result in an improvement in technique with continued education. In order to prevent critical errors in general practice, resident physicians should receive hands-on brachytherapy experience under attending guidance.

The involvement of medical students in clinical care is often approached by patients with apprehension. Although all medical student involvement is overseen by attending physicians, there is a general concern of medical students causing unforeseen side effects or outcomes. Our series demonstrated that medical student involvement did not have an adverse impact on brachytherapy outcomes. Medical students should be encouraged to actively participate in clinics and procedures under direct supervision.

Prostate cancer was associated with one of the highest cancer health care costs in the United States in 2010 [14]. Men diagnosed with localized prostate cancer have multiple effective treatment options and as a result, cost-effectiveness has become an important driver in decision making $[15,16]$. A recent analysis by Shah et al demonstrated that high dose rate and LDR brachytherapy were less costly than IMRT without any significant differences in 5 -year biochemical control or cause-specific survival [17]. In this era of identifying costeffective treatment options for patients with localized prostate cancer, brachytherapy appears 
to be an optimal solution. Unfortunately, multiple series have demonstrated a decreasing trend in the utilization of prostate brachytherapy $[1,18]$. This has been attributed to early adoption of expensive new technology as well as increased active surveillance in these patients. Furthermore, many residency programs do not perform LDR brachytherapy and residents are forced to go rotate at outside institutions to complete residency requirements. A recent analysis of radiation oncology resident procedure logs demonstrated a $25 \%$ decrease in the mean number of interstitial procedures completed from 2006-2007 to 2010-2011 [2]. Maintaining high-quality resident training in brachytherapy is vital in this era of health care reform. Given the cost-effectiveness of this modality, further consideration for dedicated brachytherapy training during a radiation oncology training residency should be considered.

The importance of case volume and treatment outcomes is well-recognized. Chen et al used the SEER-Medicare database to identify the impact of treatment volume on outcomes following brachytherapy. In their analysis, men treated at high volume facilities had lower rates of complications, recurrence rates, and prostate cancer specific mortality [19]. Other series have demonstrated an improvement in outcomes with increasing provider and center experience suggesting a learning curve in brachytherapy procedures [20,21]. In a patterns of care study for cervical cancer, Eifel et al found that smaller treatment centers were more likely to inadequately dose point A during intracavitary implants [22]. These findings are not surprising, multiple surgical series have demonstrated a direct correlation between case volume and outcomes in patients undergoing surgical procedures [23, 24]. Training experienced physicians is an important aspect of residency, and brachytherapy training should continue to be an important aspect of radiation oncology training.

Multiple surgical series have examined the impact of trainee involvement on postoperative complications with conflicting results [3, 25-27]. These conflicting reports are often believed to be secondary to differences in institutional reporting and complexity of surgical procedures. For example, oncologic surgeries are generally considered far more complex and have increased peri-operative morbidity and mortality versus non-oncologic procedures. Attending physician supervision is a well-recognized predictor of surgical outcomes. In a series by Fallon et al, attending physician involvement in surgical procedures was correlated with decreased complications and mortality rates [28]. In our series, all procedures were conducted under the supervision of a radiation oncologist that was well-experienced in brachytherapy. Our findings confirm that under experienced guidance, brachytherapy is an effective and well-tolerated procedure.

A limitation of our study is its retrospective nature and as a result, confounding variables cannot be entirely excluded. Furthermore, this series cannot account for details of resident and medical student involvement in each case. Although trainees routinely actively participate in procedures, we were unable to identify cases where trainee involvement was more limited. In addition, identifying differences in biochemical failure and survival will likely require further follow-up. Finally, we do not present data on operative time, blood loss and other descriptive variables. These may ultimately be impacted by trainee involvement due to a lack of trainee expertise in placing needles. 


\section{Conclusions}

Patients undergoing surgical procedures commonly express concern regarding trainee involvement and the impact on treatment outcomes. Multiple surgical series have reported conflicting results regarding the impact of trainee involvement on surgical outcomes [3, 25-27]. To our knowledge, this is the first analysis examining the impact of fellow, resident, or medical student participation on dosimetric or clinical outcomes in the radiation oncology setting. Per our institutional data, trainee involvement does not impact patient outcomes.

\section{Acknowledgments}

This publication was supported by grant number P30 CA006927 from the National Cancer Institute, NIH. Its contents are solely the responsibility of the authors and do not necessarily represent the official views of the National Cancer Institute or the National Institutes of Health. This publication was also supported in part by a grant from Varian Medical Systems, Inc. Its contents are solely the responsibility of the authors and do not necessarily represent the official views of Varian Medical Systems, Inc.

\section{Works Cited}

1. Martin JM, Handorf EA, Kutikov A, et al. The rise and fall of prostate brachytherapy: use of brachytherapy for the treatment of localized prostate cancer in the National Cancer Data Base. Cancer. 2014; 120:2114-2121. [PubMed: 24737481]

2. Compton JJ, Gaspar LE, Shrieve DC, et al. Resident-reported brachytherapy experience in ACGME-accredited radiation oncology training programs. Brachytherapy. 2013; 12:622-627. [PubMed: 23973187]

3. Castleberry AW, Clary BM, Migaly J, et al. Resident education in the era of patient safety: a nationwide analysis of outcomes and complications in resident-assisted oncologic surgery. Ann Surg Oncol. 2013; 20:3715-3724. [PubMed: 23864306]

4. Guo LR, Chu MW, Tong MZ, et al. Does the trainee's level of experience impact on patient safety and clinical outcomes in coronary artery bypass surgery? J Card Surg. 2008; 23:1-5. [PubMed: 18290878]

5. Mohler JL, Kantoff PW, Armstrong AJ, et al. Prostate Cancer, Version 1.2014 Featured Updates to the NCCN Guidelines. Journal of the National Comprehensive Cancer Network. 2013; 11:14711479. [PubMed: 24335682]

6. Davis BJ, Horwitz EM, Lee WR, et al. American Brachytherapy Society consensus guidelines for transrectal ultrasound-guided permanent prostate brachytherapy. Brachytherapy. 2012; 11:6-19. [PubMed: 22265434]

7. Sharma NK, Cohen RJ, Eade TN, et al. An intraoperative real-time sleeved seed technique for permanent prostate brachytherapy. Brachytherapy. 2010; 9:126-130. [PubMed: 19850534]

8. Lubbe W, Cohen R, Sharma N, et al. Biochemical and clinical experience with real-time intraoperatively planned permanent prostate brachytherapy. Brachytherapy. 2012; 11:209-213. [PubMed: 21727033]

9. Roach M 3rd, Hanks G, Thames H Jr, et al. Defining biochemical failure following radiotherapy with or without hormonal therapy in men with clinically localized prostate cancer: recommendations of the RTOG-ASTRO Phoenix Consensus Conference. Int J Radiat Oncol Biol Phys. 2006; 65:965-974. [PubMed: 16798415]

10. Murphy CT, Heller S, Ruth K, et al. Evaluating toxicity from definitive radiation therapy for prostate cancer in men with inflammatory bowel disease: Patient selection and dosimetric parameters with modern treatment techniques. Pract Radiat Oncol. 2015; 5:e215-222. [PubMed: 25424586]

11. Pollack A, Hanlon AL, Horwitz EM, et al. Dosimetry and preliminary acute toxicity in the first 100 men treated for prostate cancer on a randomized hypofractionation dose escalation trial. Int $\mathbf{J}$ Radiat Oncol Biol Phys. 2006; 64:518-526. [PubMed: 16242256] 
12. Stock RG, Stone NN, Tabert A, et al. A dose-response study for I-125 prostate implants. Int J Radiat Oncol Biol Phys. 1998; 41:101-108. [PubMed: 9588923]

13. Potters L, Cao Y, Calugaru E, et al. A comprehensive review of CT-based dosimetry parameters and biochemical control in patients treated with permanent prostate brachytherapy. Int J Radiat Oncol Biol Phys. 2001; 50:605-614. [PubMed: 11395226]

14. Mariotto AB, Yabroff KR, Shao Y, et al. Projections of the cost of cancer care in the United States: 2010-2020. J Natl Cancer Inst. 2011; 103:117-128. [PubMed: 21228314]

15. D'Amico AV, Whittington R, Malkowicz SB, et al. Biochemical outcome after radical prostatectomy, external beam radiation therapy, or interstitial radiation therapy for clinically localized prostate cancer. JAMA. 1998; 280:969-974. [PubMed: 9749478]

16. Grimm P, Billiet I, Bostwick D, et al. Comparative analysis of prostate-specific antigen free survival outcomes for patients with low, intermediate and high risk prostate cancer treatment by radical therapy. Results from the Prostate Cancer Results Study Group. BJU Int. 2012; 109(Suppl 1):22-29. [PubMed: 22239226]

17. Shah C, Lanni TB Jr, Ghilezan MI, et al. Brachytherapy provides comparable outcomes and improved cost-effectiveness in the treatment of low/intermediate prostate cancer. Brachytherapy. 2012; 11:441-445. [PubMed: 22728157]

18. Mahmood U, Pugh T, Frank S, et al. Declining use of brachytherapy for the treatment of prostate cancer. Brachytherapy. 2014; 13:157-162. [PubMed: 24050892]

19. Chen AB, D'Amico AV, Neville BA, et al. Provider case volume and outcomes following prostate brachytherapy. J Urol. 2009; 181:113-118. discussion 118. [PubMed: 19012905]

20. Lee WR, deGuzman AF, Bare RL, et al. Postimplant analysis of transperineal interstitial permanent prostate brachytherapy: evidence for a learning curve in the first year at a single institution. Int J Radiat Oncol Biol Phys. 2000; 46:83-88. [PubMed: 10656377]

21. Keyes M, Schellenberg D, Moravan V, et al. Decline in urinary retention incidence in 805 patients after prostate brachytherapy: the effect of learning curve? Int J Radiat Oncol Biol Phys. 2006; 64:825-834. [PubMed: 16458775]

22. Eifel PJ, Moughan J, Erickson B, et al. Patterns of radiotherapy practice for patients with carcinoma of the uterine cervix: a patterns of care study. Int J Radiat Oncol Biol Phys. 2004; 60:1144-1153. [PubMed: 15519786]

23. Birkmeyer JD, Siewers AE, Finlayson EV, et al. Hospital volume and surgical mortality in the United States. N Engl J Med. 2002; 346:1128-1137. [PubMed: 11948273]

24. Begg CB, Cramer LD, Hoskins WJ, Brennan MF. Impact of hospital volume on operative mortality for major cancer surgery. JAMA. 1998; 280:1747-1751. [PubMed: 9842949]

25. Patel SP, Gauger PG, Brown DL, et al. Resident participation does not affect surgical outcomes, despite introduction of new techniques. J Am Coll Surg. 2010; 211:540-545. [PubMed: 20801695]

26. Shaked A, Calderom I, Durst A. Safety of surgical procedures performed by residents. Arch Surg. 1991; 126:559-560. [PubMed: 2021334]

27. Doerr RJ, Yildiz I, Flint LM. Pancreaticoduodenectomy University experience and resident education. Arch Surg. 1990; 125:463-465. [PubMed: 2322112]

28. Fallon WF Jr, Wears RL, Tepas JJ 3rd. Resident supervision in the operating room: does this impact on outcome? J Trauma. 1993; 35:556-560. discussion 560-551. [PubMed: 8411279]

\section{Abbreviations}

$\begin{array}{ll}\text { ABS } & \text { American Brachytherapy Society } \\ \text { CT } & \text { Computed Tomography } \\ \text { D90 } & \text { minimum dose covering } 90 \% \text { of the prostate } \\ \text { FFBF } & \text { Freedom from biochemical failure } \\ \text { I-125 } & \text { Iodine } 125\end{array}$

Brachytherapy. Author manuscript; available in PMC 2017 March 01. 
LDR low dose rate

MRI Magnetic Resonance Imaging

NCCN National Comprehensive Cancer Network

PSA Prostate specific antigen

V100 Volume of the prostate receiving $100 \%$ of the prescription dose 

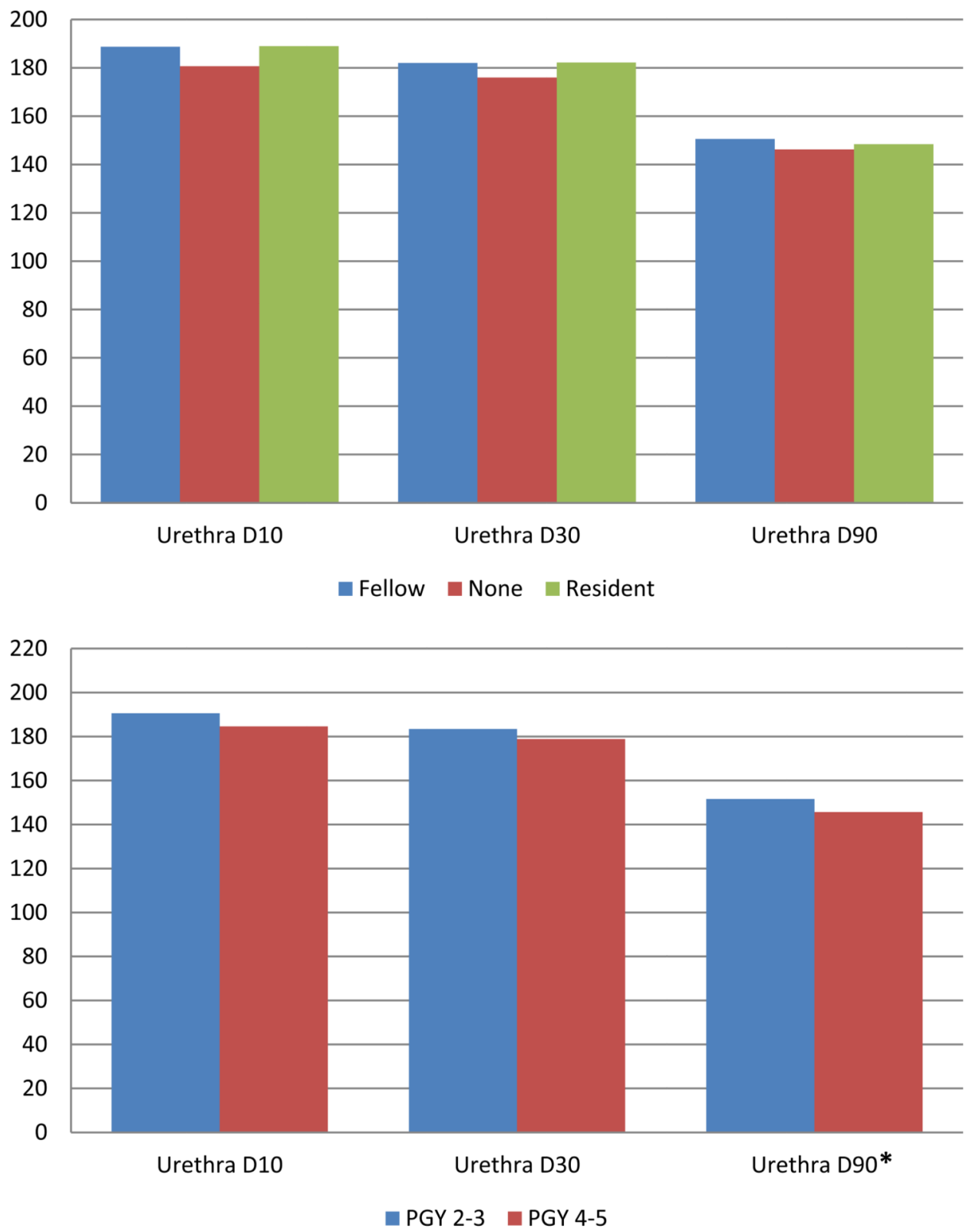


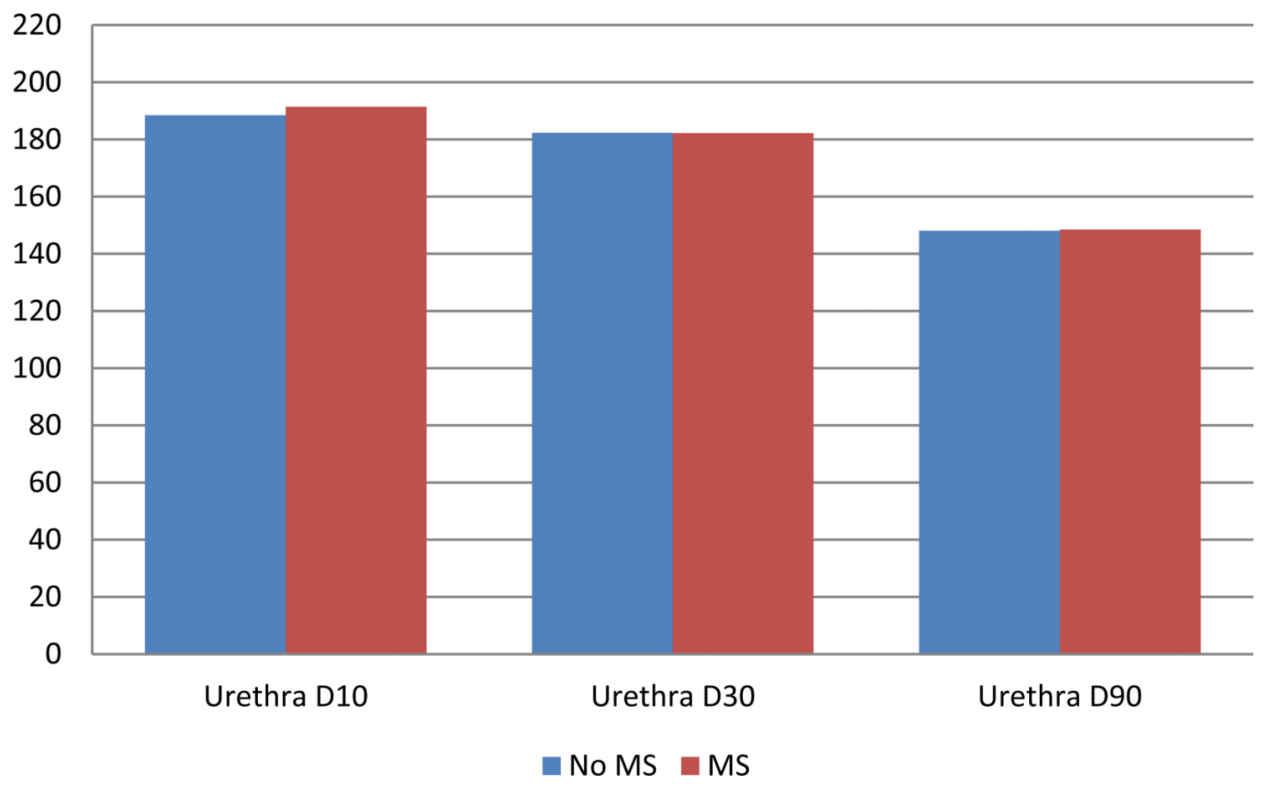

Figure 1. A, B, C: Impact of trainee involvement on urethral dosimetric endpoints

* Statistically significant difference $(\mathrm{p}<0.05)$ 


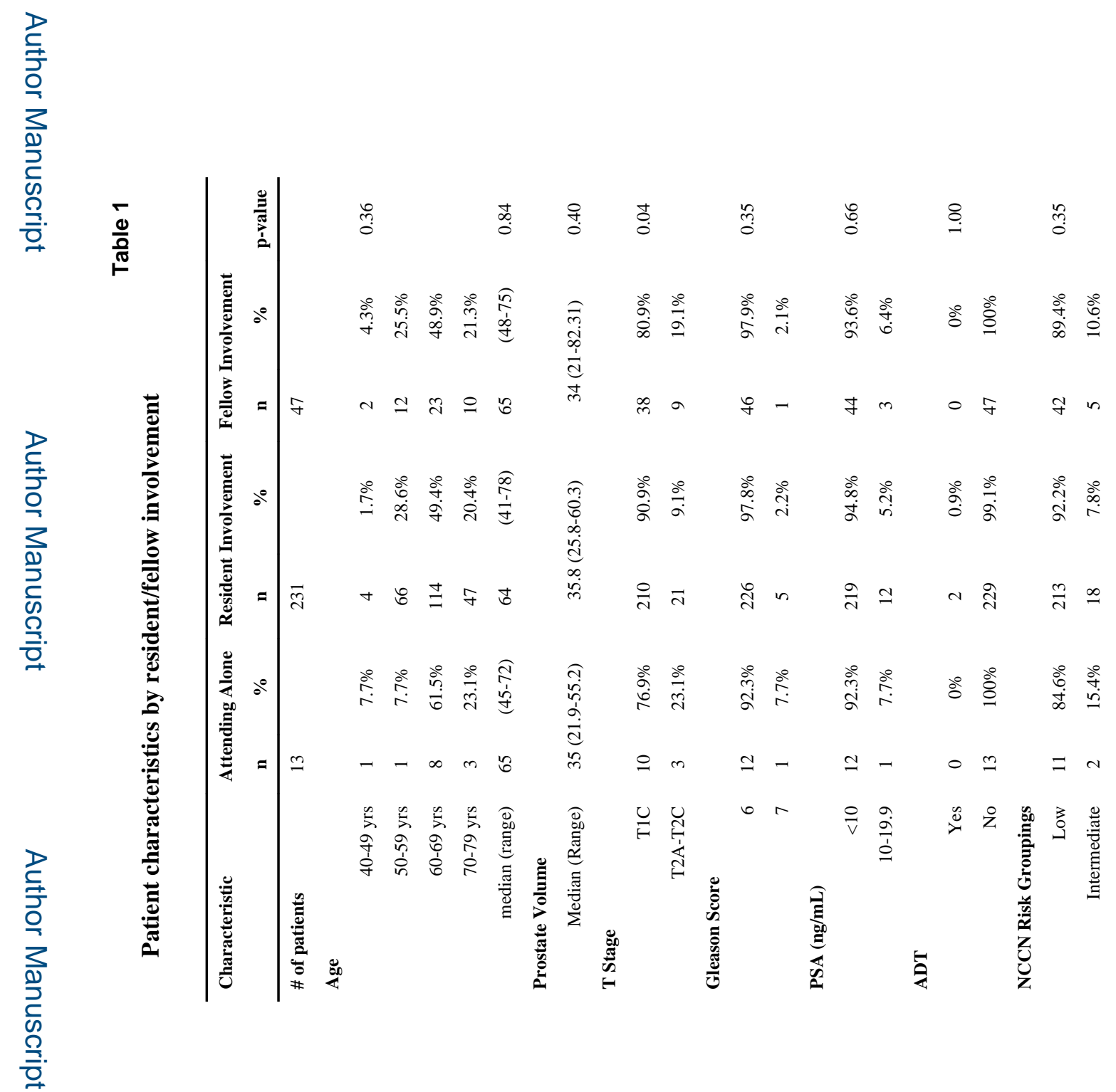



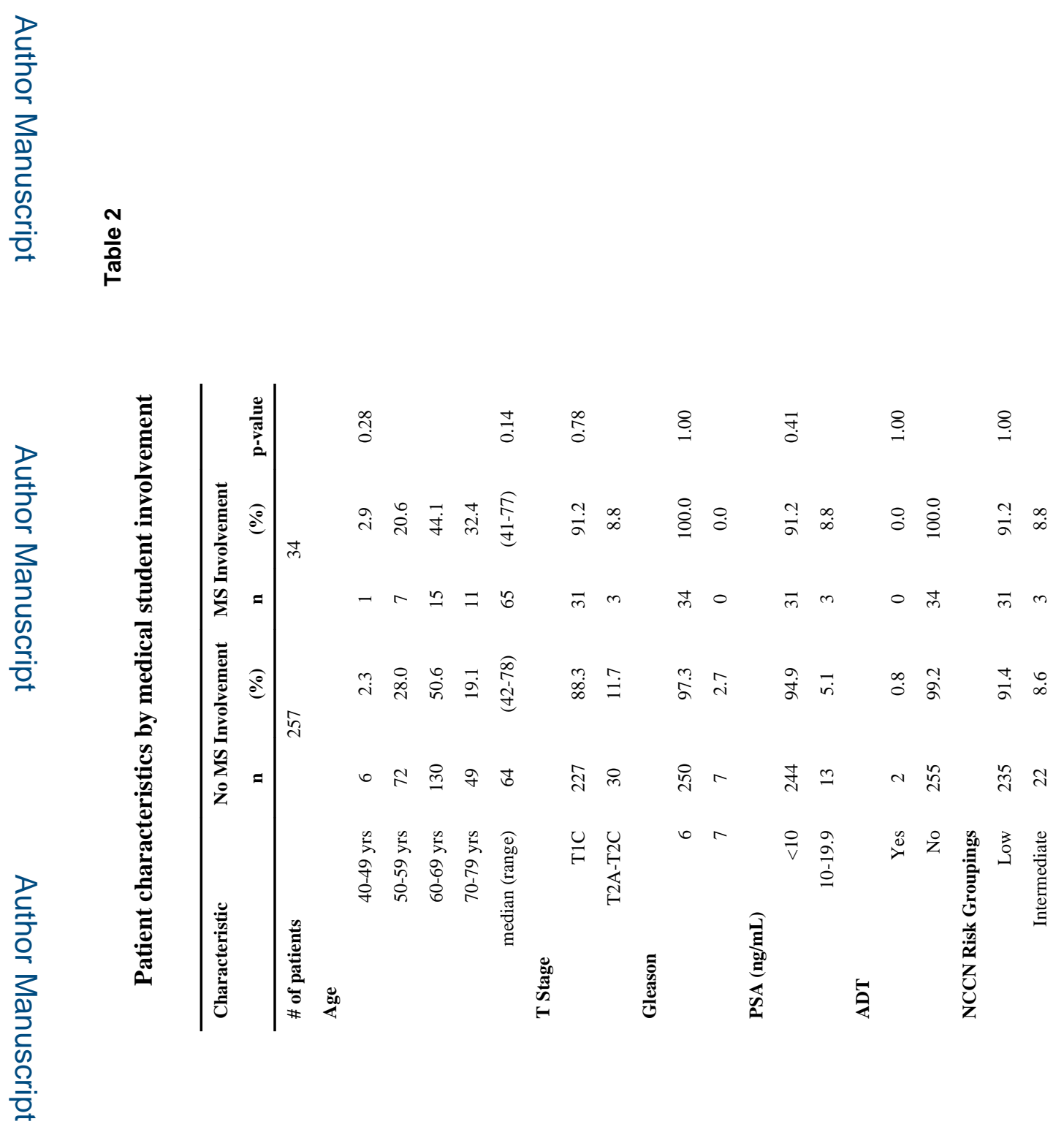

Brachytherapy. Author manuscript; available in PMC 2017 March 01. 


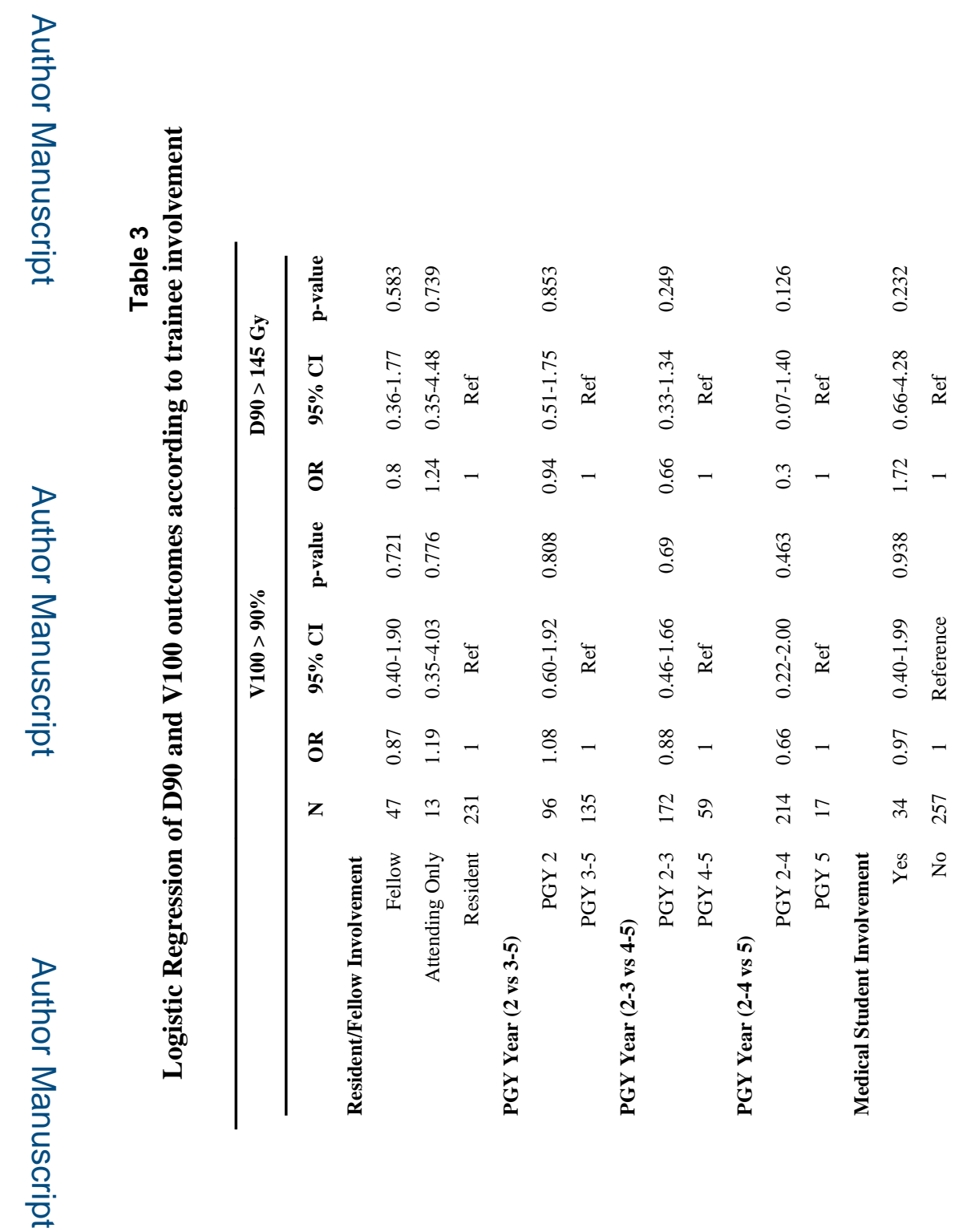

Brachytherapy. Author manuscript; available in PMC 2017 March 01. 